\title{
A Unique TAS Setup for high multiplicity events at VECC, Kolkata using $\mathrm{BaF}_{2}$ detectors
}

\author{
G. Mukherjee ${ }^{1 \mathrm{a}}$, Balaram Dey ${ }^{1}$, S. Mukhopadhyay ${ }^{1}$, Deepak Pandit ${ }^{1}$, Surajit Pal ${ }^{1}$, H. Pai $^{1 \mathrm{~b}}$ and S.R. \\ Banerjee $^{1}$ \\ ${ }^{1}$ Variable Energy Cyclotron Centre, 1/AF Bidhan Nagar, Kolkata 700064, India
}

\begin{abstract}
A granular total absorption spectrometer (TAS) has been developed at the Variable Energy Cyclotron Centre, Kolkata, India using 50 elements of $\mathrm{BaF}_{2}$ detectors and covering $4 \pi$. The advantage with such a granular setup is that one can get sum spectrum with the condition of different multiplicity hits in an event. It has been shown that one can get clean sum-peaks devoid of individual peaks with the choice of two or higher fold of multiplicity. The large granularity makes it a unique TAS setup particularly for the high multiplicity events. The set up has been tested using different radioactive sources with one, two or multiple $\gamma$ rays in cascade. The set up is ready to be used online.
\end{abstract}

\section{Introduction}

A Total Absorption Spectrometer (TAS) is a setup to measure the total energy of all the $\gamma$ rays emitted in an event. Such a setup is very useful for the measurement of $\beta$-decay feeding intensity free from pandemonium effect [1 - 4]. Accurate measurement of $\beta$-decay strength function is necessary for both in the basic understanding of the $\beta$-decay process, in particular, the Gammow-Teller decay in the vicinity of closed shell nuclei and also in the applied field for the estimation of decay heat [5]. Information about the level properties and the decay processes in $\alpha$ and $\gamma$ spectroscopy are obtained from direct measurements of decay spectra. For $\beta$-decay, it is somewhat complicated as in this case the $\beta$-decay spectrum is continuous in nature. Therefore, information about the decay probability of the $\beta$-decay is obtained indirectly from the measurement of the intensity balance of the $\gamma$-rays from the levels populated in the $\beta$-decay transitions. In these cases the gamma detector array with high resolution HPGe detectors are used for the detection of $\gamma$ rays to measure the feeding intensities. In these high resolution measurements, there are two main difficulties: low detection efficiency and the fragmentation of gamma intensity. The efficiency of the HPGe detectors is very low, both for the high and low energy $\gamma$ rays as the photo-peak efficiency is low for the high energy $\gamma$ rays and for the low energy $\gamma$ rays, the absorption in the casing and dead layers become more important. Therefore, many weak transitions remain unobserved in the high resolution measurements and, hence, the feeding intensity would be wrongly deduced. In these cases, much of the $\beta$-feeding are not observed and then incorrectly assigned to higher feeding for the low lying levels or to the ground state. This is what is

\footnotetext{
${ }^{\mathrm{a}}$ Corresponding author: gopal@vecc.gov.in

${ }^{\mathrm{b}}$ Present address: Institut für Kernphysik, Technische

Universităt Darmstadt, GERMANY.
} 
termed as Pandemonium effect. The solution to this experimental problem is to adopt a different approach to the measurement using a TAS which is sensitive to the $\beta$-population of the nuclear levels instead of individual $\gamma$ rays. In the ideal case, TAS has detection efficiency of $100 \%$. Instead of having individual $\gamma$ peaks, a sum energy peak corresponding to the total energy of the gamma cascade following the $\beta$-decay is measured. The intensity of this sum-peak, ideally, gives direct information about the levels fed in the decay. Total absorption spectrometers are, usually, constructed using large scintillator detectors covering $4 \pi$ of the solid angle around the $\beta$-emitting source. Two main criteria for a TAS set up are $4 \pi$ coverage and knowledge of the detector response. A few TAS set up that exist in the world are mostly constructed from large $\mathrm{NaI}(\mathrm{Tl})$ detectors because of its high efficiency and low cost. One of the deficiencies of such large volume setups is that it cannot distinguish a single $\gamma$ ray from the cascade of $\gamma$-rays whose sum is close to that of a single $\gamma$-ray energy. At VECC a TAS setup has been developed using closely packed 50 numbers of smaller volume $\mathrm{BaF}_{2}$ detectors which can be used to overcome the above deficiency. In this unique modular setup multiplicity gated sum energy is obtained to study the beta decay strength function for physics interest as well as for providing input for the estimation of decay heat of the reactors, particularly for the nuclei for which TAS measurements are reported to be essential [6].

\section{The TAS set up at VECC}
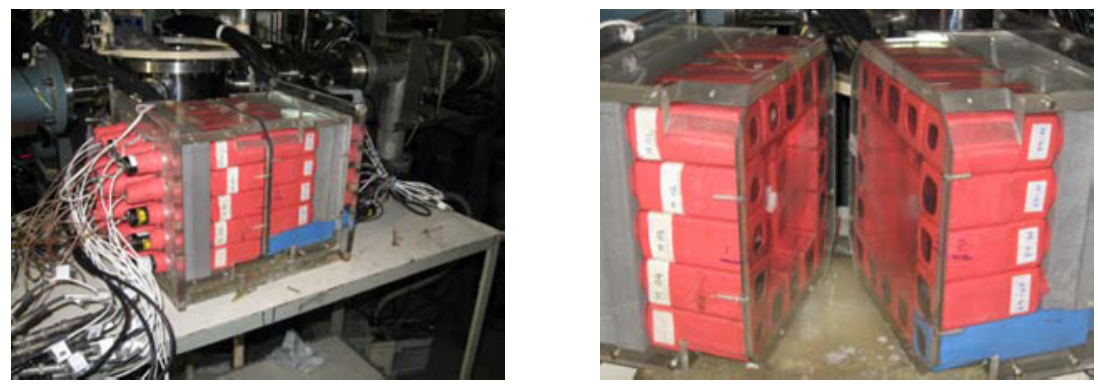

Figure 1. (Left) The closed configuration and (right) two halves of the TAS array at VECC are shown.

The TAS set up at VECC has been shown in Figure 1. The set up consists of 50 elements of $\mathrm{BaF}_{2}$ detectors. The length of each detector is $5 \mathrm{~cm}$ with a cross sectional area of $3.5 \times 3.5 \mathrm{~cm}^{2}$. The energy resolution of the detectors was about $8 \%$. Other details about the detectors and their characterization can be found in Ref. [7]. The full array (Figure 1, left) consists of two blocks having 25 detectors in each one of them. The detectors in each block are arranged in castle type geometry (Figure 1, right). The blocks are brought together for a closed $4 \pi$ configuration. The radiation source can be kept inside and at the middle of the array. The large granularity of $\mathrm{BaF}_{2}$ detectors make the present set up unique. The advantages of the large granularity have been depicted in subsequent sections. Simulations (in GEANT 3 platform) were also performed [8] for two other geometries with the above detectors and also with similar but longer $(35 \mathrm{~cm})$ detectors [9].

\section{Measurements with radioactive sources}

The set up has been tested using radioactive sources with known $\gamma$ rays. One-, two-, three- and multi- $\gamma$ line sources ${ }^{137} \mathrm{Cs},{ }^{60} \mathrm{Co},{ }^{22} \mathrm{Na}$ and ${ }^{152} \mathrm{Eu}$, respectively, were used for characterization and testing of the TAS setup. A ${ }^{137} \mathrm{Cs}$ spectrum obtained from a single individual $\mathrm{BaF}_{2}$ crystal is shown in Figure 2 . Apart from the characteristic $662-\mathrm{keV}$ peak, other peaks are observed in this spectrum above $1.5 \mathrm{MeV}$ are due to the inherent $\alpha$-decays in the $\mathrm{BaF}_{2}$ crystal itself, the intensity of which are very small (two orders of magnitude less) compared to the 662-keV $\gamma$-peak. These fixed energy peaks help to check on the stability of the energy gain during the experiment. 


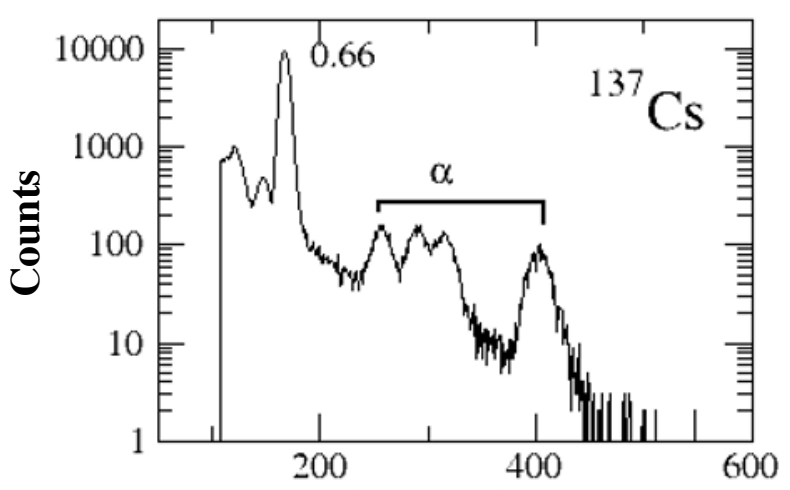

Channel No.

Figure 2. Raw spectrum of ${ }^{137} \mathrm{Cs}$ source obtained from a single $\mathrm{BaF}_{2}$ detector.

It is very important to separate the sum peaks from the individual peaks to get a clean sum spectrum. That can be achieved in the offline analysis by incorporating the condition of multiplicity hit in obtaining the sum energy, in our modular spectrometer. Energy calibration and gain matching of the crystals were done before obtaining the sum spectrum. A multiplicity spectrum, defined by the number of crystals hit in an event, was generated and the sum spectra were obtained using different condition of multiplicity (M). Sum spectra for ${ }^{60} \mathrm{Co}$ and ${ }^{22} \mathrm{Na}$ sources are shown in Figure 3 for different conditions of $\mathrm{M}$.
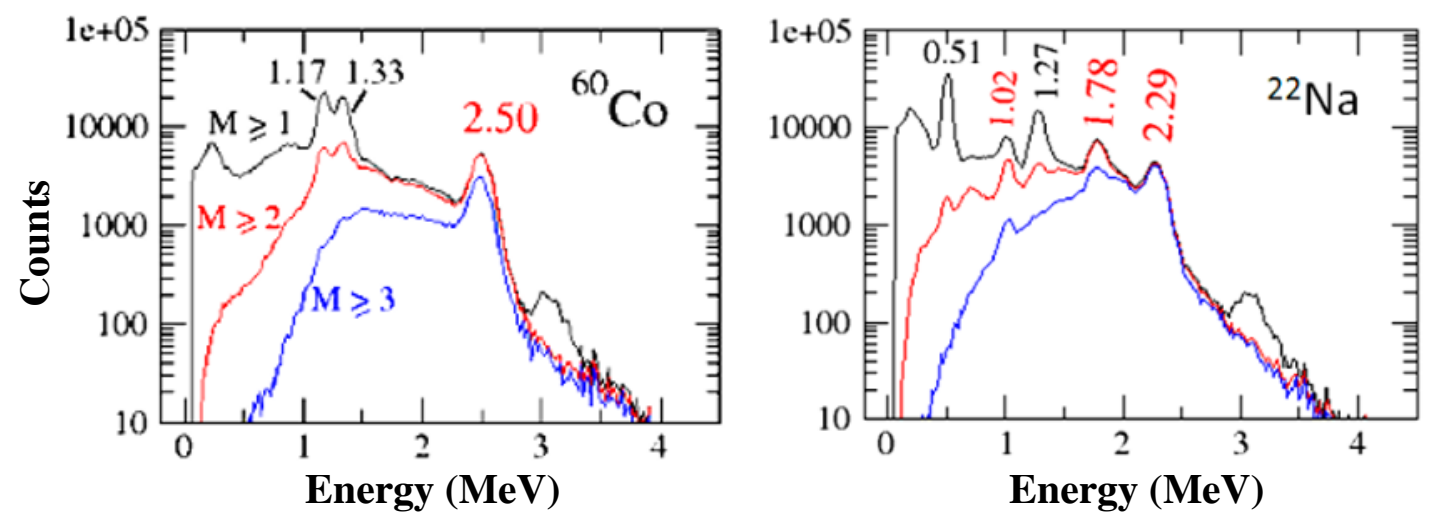

Figure 3. Sum spectra of ${ }^{60} \mathrm{Co}$ (left) and ${ }^{22} \mathrm{Na}$ sources obtained with different multiplicity (M) conditions. In each panel, top one (black) is for $M \geq 1$, the middle one (red) is for $M \geq 2$ and bottom one (blue) is for $M \geq 3$.

It can be seen from the above figure that the individual peaks (1.17 and $1.33 \mathrm{MeV}$ for ${ }^{60} \mathrm{Co}$ and 0.51 and $1.27 \mathrm{MeV}$ for ${ }^{22} \mathrm{Na}$ ) and the sum peaks (2.5 MeV for ${ }^{60} \mathrm{Co}$ and $1.02,1.78$ and $2.29 \mathrm{MeV}$ for ${ }^{22} \mathrm{Na}$ ) are clearly seen in the sum spectra with $M \geq 1$. For $M \geq 2$, intensities of the individual peaks reduced significantly with no loss of intensities for the sum peaks. The individual peaks still remains, albeit less intensity, in this spectrum because of the cross talk in the array. When the multiplicity increases further $(M \geq 3)$, the individual peaks completely vanish from the spectra leaving only the sum peaks with a slight reduction in intensity for the peaks which are the sum of two individual $\gamma$ rays (e.g 2.5 $\mathrm{MeV}$ in ${ }^{60} \mathrm{Co}$ and $1.78 \mathrm{MeV}$ in ${ }^{22} \mathrm{Na}$ ). For the peaks which are sum of more than two $\gamma$ rays (e.g 2.29 $\mathrm{MeV}$ in ${ }^{22} \mathrm{Na}$ ), there is no loss of intensity for $\mathrm{M} \geq 3$. This way one can easily identify the sum peaks from the individual ones and get a clean sum peak spectrum. Moreover, one can identify the number of individual $\gamma$ rays contributes to a particular sum peak. The relative intensities of the sum peaks would then correspond to the relative feeding intensity of the daughter states corresponding to that sum peak. 


\subsection{Sum spectrum and beta feeding in ${ }^{152} \mathrm{Eu}$}

Several $\gamma$ rays are known in a ${ }^{152}$ Eu source, the ground state of which decays to ${ }^{152} \mathrm{Gd}$ and ${ }^{152} \mathrm{Sm}$ by $\beta^{-}$ and $\varepsilon$ decays with branching ratio of $28 \%$ and $72 \%$, respectively [10]. The beta feeding from the parent is fragmented in several daughter states with most of the feeding take place in the three states at $1.09 \mathrm{MeV}$ (21.7\%), $1.23 \mathrm{MeV}$ (17.4\%) and $1.53 \mathrm{MeV}$ (24.9\%) in ${ }^{152} \mathrm{Sm}$. The sum spectrum for ${ }^{152} \mathrm{Eu}$ source, obtained in our set up, is shown in Figure 4.

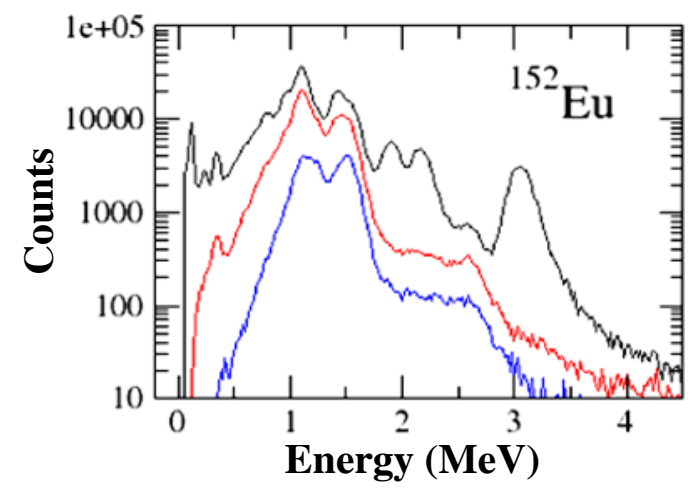

Figure 4. Same as Figure 3 but for ${ }^{152}$ Eu source.

It can be seen from this figure that three peaks remain in the sum spectrum for the multiplicity condition of $\mathrm{M} \geq 3$, at energies close to $1.1 \mathrm{MeV}, 1.2 \mathrm{MeV}$ and $1.5 \mathrm{MeV}$. These are exactly the energies of the three excited states in ${ }^{152} \mathrm{Sm}$ in which most of the beta decay feeding occurs from

${ }^{152} \mathrm{Eu}$. The relative intensities of the peaks closely match with the relative feeding intensity of the states. To get the absolute feeding intensity, one has to do a proper normalization. Therefore, it shows that it is possible to get the feeding intensity directly from the sum peaks.

\section{Summary}

A unique modular TAS set up has been developed at the Variable Energy Cyclotron Centre, Kolkata, India using 50 elements of $\mathrm{BaF}_{2}$ detectors. In this setup, it is possible to obtain sum spectrum gated by different multiplicity hit. The setup has been successfully tested using four different sources which emit single to multiple $\gamma$ rays. More involved analysis with proper simulation is needed to obtain feeding intensities of all the levels.

\section{References}

1. P. Sarriguren et al., Nucl. Phys. A658, 13 (1999)

2. A. Algora, Nucl. Phys. A654, 727 (1999)

3. B. Rubio et al., J. Phys. G : Nucl. Part. Phys. 31, S1477 (2005)

4. M. Karny et al., Nucl. Inst. Meth. Phys. Res. B126, 411 (1997)

5. A. Algora et al., Phys. Rev. Lett. 105, 202501 (2010); J. Korean Phys. Soc. 59, 1479s (2011)

6. M. Gupta et al., INDC (NDS)-0577 Tech. Doc. IAEA, May 2010

7. Deepak Pandit et al., Nucl. Inst. Meth. Phys. Res. A624 148 (2010)

8. G. Mukherjee et al., DAE Symp. on Nucl. Phys. 55, 708 (2010), http://www.sympnp.org/proceedings/

9. S. Mukhopadhyay et al., Nucl. Inst. Meth. Phys. Res. A582 603 (2007)

10. http://www.nndc.bnl.gov/nudat2/decaysearchdirect.jsp?nuc=152EU\&unc=nds 